\title{
Clinical spectrum and challenges of Primary Immunodeficiency disorders in Egyptian children
}

\author{
Nermeen M. Galal \\ Department of Pediatrics, Faculty of Medicine, Cairo University, Egypt; \\ Nermeen.galal@kasralainy.edu.eg, ORCID ID: https://orcid.org/0000-0002-6595-3641 \\ * Correspondence: Nermeen.galal@kasralainy.edu.eg \\ Received: 5/10/2020; Accepted: 25/10/2020; Published online: 25/12/2020.
}

\begin{abstract}
:
Primary Immunodeficiency Disorders (PID) are inherent defects of the immune system that may present in infants and children. The main presentations include increased susceptibility to infections (a narrow or broad spectrum of pathogens), autoimmunity, autoinflammation, allergy and/or malignancy. PIDs are increasingly diagnosed and may present with different phenotypes that range from subtle to severe ones. They are classified into ten major categories according to the International Union of Immunological Societies (IUIS). Autosomal recessive disorders are noticeably more common because of the high consanguinity rates in the society. The spectrum of PID is highlighted with a brief walk through the challenges of establishing a PID service in a resource limited setting like catching up with the rapidly changing field, provision of state of the art diagnostic services and actual provision of medical care to PID patients. Several opportunities for growth of the field and overcoming obstacles are discussed.

Level of Evidence of Work: IV (1).

Keywords: Primary Immunodeficiency Disorders; Recurrent Infections; Spectrum; Egypt Abbreviations: IUIS: International Union of Immunological Societies; PID: Primary Immunodeficiency Disorders; RAG: recombination-activating gene; RFX; regulatory factor X5; RFXANK: regulatory factor X associated ankyrin containing protein; SCID: Severe Combined Immunodeficiency Disorder; T-B-SCID: T cell-negative, B cell negative Severe Combined Immunodeficiency Disorder.
\end{abstract}

\section{Introduction}

Primary Immunodeficiency Disorders (PID) are inherent defects of the immune system that may affect the interplay of different cells and cytokines in different organs. The main presentations include increased susceptibility to infections (a narrow or broad spectrum of pathogens), autoimmunity, autoinflammation, allergy and/or malignancy.

\section{Clinical spectrum}

PID disorders include over 406 different disorders and are classified into ten major categories according to the International Union of Immunological Societies (IUIS); immunodeficiencies with cellular or humoral defects, combined immunodeficiency with syndromic features, predominantly antibody deficiencies, disorders of immune dysregulation, congenital defects of phagocytes, defects of innate immunity, autoinflammatory disorders, complement defects, bone marrow failure and phenocopies of PID (2).

The Primary Immunodeficiency Clinic -Cairo University was founded in 2004 by Professor Aisha Elmarsafy, an internationally acclaimed figure in the PID field and the lead for PID services. The unit has a clinical division and a laboratory division run by colleagues in the Clinical Pathology Department, Faculty of Medicine, Cairo University who over the years have managed to evolve rapidly to cope with changes in the dynamic field expanding the panels offered by the laboratory. Because of the high rates of consanguinity in Egypt, autosomal recessive disorders prevail and lead to a different distribution of PID categories compared to Europe, North 
and Latin America and a peculiar distribution of major PID categories, particularly more combined immunodeficiencies than antibody disorders as is the situation in similar countries (3).

Since 2010, over 10,000 children have been referred to the clinic for assessments and in the interval from 2010-2014 four hundred and sixty-six children were identified and documented with proved PID disorders. One hundred twenty children were diagnosed with probable PID (not matching specific disease criteria or not having enough evidence to support the diagnosis yet having clinical manifestations highly suggestive of PID). T cell/combined disorders constituted $30 \%$, followed by predominantly antibody disorders (19\%), syndromic combined disorders (16\%), phagocytic defects of number or function (13\%), immune dysregulation (11\%), autoinflammatory disorders (9\%), complement deficiency (1\%) and defects of innate immunity (1\%).

Severe Combined Immunodeficiency Disorder (SCID), a neonatal emergency, remains one of the most encountered presentations of combined Immunodeficiency. The early diagnosis dramatically affects the outcome of the disease by allowing bone marrow transplantation at an early age, and providing prenatal diagnosis and genetic counseling for families with a positive history. A nationwide neonatal screening program will definitely help identify those infants at an early age and direct them to therapy. For the time being targeted screening for high risk families is done. Screening high risk families revealed a prevalence of SCID, with $70 \%$ T-BSCID patients and $30 \%$ T-B+ SCID patients, as the most common PID picked up through screening. Lower respiratory tract infections, persistent fungal infections and lymphopenia were the most significant warning signs for diagnosing PID in screened newborns.

Study of the molecular defects in SCID led to identification of novel mutations in patients with recombination-activating gene (RAG) mutations (4). Major Histocompatibility Complex Deficiency, another autosomal recessive combined PID revealed a prevalence of regulatory factor $\mathrm{X}$ associated ankyrin containing protein (RFXANK) and regulatory factor X5 (RFX5) gene mutations with previously unreported mutations (5) emphasizing the peculiarity of those disorders in this part of the world.

\section{Challenges}

The field of PID is challenged by many obstacles, it is a dynamically changing field and diagnosis requires high quality state of the art laboratory services that are very costly. Identification of genetic defects is very crucial for several reasons as diagnosis of atypical presentations, carrier identification, offering prenatal diagnosis and genetic counselling for affected families and establishing prognosis. To date prenatal diagnosis has been offered to more than twenty families with different PID disorders like SCID, chronic granulomatous disease and early onset inflammatory bowel disease in collaboration with the department of Obstetrics and Gynecology (6).

Another obstacle is the lack of public and professional awareness about those disorders which leads to under diagnosis/ late diagnosis with a very complicated disease course. This has been partly improved by increased physician awareness but still needs mass campaigns for public awareness, avoiding consanguineous marriages, withholding live vaccines and seeking care early in case of suspicion.

Access to treatment is a very challenging aspect of PID service provision in Egypt, for example the monthly replacement Intravenous Immunoglobulins for patients with antibody disorders, the HLA typing tests for family members to assess feasibility for transplantation and if fortunate enough to find a matched donor and the timely finding of funding bodies to cover transplantation costs. Care for patients with PID should primarily be based on a multidisciplinary approach with input from different pediatric subspecialties as well as other disciplines as radiology, dermatology, pulmonology, orthopedics and neurosurgery along with other specialties, a process that requires constant integration for holistic patient care provision.

Transition to adult care is another area that still needs improvement to facilitate service provision for our growing children who are sometimes burdened by social stigma and need scholastic aid for missed school days. The set-up of patient societies may ease some of the problems encountered by those patients. 


\section{Opportunities}

Since it is very complicated to set up a PID service center, the establishment of multiple satellites for patient identification, referral and follow up after diagnosis has been a successful model. Networking is done with different centers across Egypt to ease patients' service provision. Educational workshops are done in liaison with other specialties and trainees from neighboring countries as Libya, Sudan and Yemen also help set up similar practices in their respective countries.

Collaboration between local centers also provides complementary laboratory panels, exchange of expertise and nation-wide surveillance as with Polio virus detection in Primary Immunodeficiency patients for Polio virus eradication currently conducted with Ain shams and Alexandria Universities as patients with PID may constitute a reservoir shedding virus in the community $(7,8)$. Patient documentation in different centers can act as a nidus for a longawaited national registry establishment which will enhance effectiveness of services for involved parties and help better identify disease patterns/outcomes.

Collaboration with different centers in The European Society of Immunodeficiency Disorders (ESID) and the African Society of Immunodeficiency Disorders (ASID) allows physician education, clinical research collaboration and guideline generation to ease diagnosis and management for PID children.

\section{Conclusion}

Egypt and North Africa present a very rich field for the study of autosomal recessive PID disorders which is very vital to characterize those diseases. Although increasing numbers of PID patients and disorders are diagnosed in the area, they still represent the tip of the iceberg of undiagnosed ailments due to unawareness or simply having subtle phenotypes.

In this rapidly changing world, primary immunodeficiency disorders continue to progress and the science remains dictated by the different patients' stories and struggles they so bravely continue to face and hopefully conquer one day.

\section{Author Contributions:}

The author searched medical literature, databases, conceptualized, drafted initial manuscript and reviewed the final manuscript. The author have read and agreed to the published version of the manuscript.

\section{FUNDING}

The author declares there was no extramural funding provided for this study.

\section{CONFLICT OF INTEREST}

The author declares no conflict of interest in connection with the reported study and is responsible for its veracity.

\section{References}

1. S. Tenny, M. Varacallo, Evidence Based Medicine. (StatPearls Publishing; Treasure Island (FL), 2020; https://www.ncbi.nlm.nih.gov/books/NBK470182/).

2. A. Bousfiha, L. Jeddane, C. Picard, W. Al-Herz, F. Ailal, T. Chatila, C. Cunningham-Rundles, A. Etzioni, J. L. Franco, S. M. Holland, C. Klein, T. Morio, H. D. Ochs, E. Oksenhendler, J. Puck, T. R. Torgerson, J.-L. Casanova, K. E. Sullivan, S. G. Tangye, Human Inborn Errors of Immunity: 2019 Update of the IUIS Phenotypical Classification. J. Clin. Immunol. 40, 66-81 (2020).

3. M.-R. Barbouche, N. Galal, I. Ben-Mustapha, L. Jeddane, F. Mellouli, F. Ailal, M. Bejaoui, J. Boutros, A. Marsafy, A. A. Bousfiha, Primary immunodeficiencies in highly consanguineous North African populations: Primary immunodeficiencies and consanguinity. Ann. N. Y. Acad. Sci. 1238, 42-52 (2011).

4. S. Meshaal, R. El Hawary, M. Elsharkawy, R. K. Mousa, R. J. Farid, D. Abd Elaziz, R. Alkady, N. Galal, M. J. Massaad, J. Boutros, A. Elmarsafy, Mutations in Recombination 
Activating Gene 1 and 2 in patients with severe combined immunodeficiency disorders in Egypt. Clin. Immunol. 158, 167-173 (2015).

5. R. E. El Hawary, A. A. Mauracher, S. S. Meshaal, A. Eldash, D. S. Abd Elaziz, R. Alkady, S. Lotfy, L. Opitz, N. M. Galal, J. A. Boutros, J. Pachlopnik Schmid, A. M. Elmarsafy, MHC-II Deficiency Among Egyptians: Novel Mutations and Unique Phenotypes. J. Allergy Clin. Immunol. Pract. 7, 856-863 (2019).

6. R. E. El Hawary, S. S. Meshaal, D. S. Abd Elaziz, M. A. Elsharkawy, R. S. Alkady, S. Lotfy, A. El-Sheikhah, A. Hassan, N. M. Galal, J. A. Boutros, A. M. Elmarsafy, Genetic Counseling in Primary Immunodeficiency Disorders: An Emerging Experience in Egypt. Mol. Diagn. Ther. 21, 677-684 (2017).

7. Z. A. El Sayed, O. Mach, Poliovirus Excretion among Persons with Primary Immune Deficiency Disorders: Summary of Data from Enhanced Poliovirus Surveillance in Egypt, 2011-2014. J. Vaccines Vaccin. 7 (2016), doi:10.4172/2157-7560.1000331.

8. N. M. Galal, S. Meshaal, R. ElHawary, E. Nasr, L. Bassiouni, H. Ashghar, N. H. Farag, O. Mach, C. Burns, J. Iber, Q. Chen, A. ElMarsafy, Poliovirus excretion following vaccination with live poliovirus vaccine in patients with primary immunodeficiency disorders: clinicians' perspectives in the endgame plan for polio eradication. BMC Res. Notes. 11, 717 (2018).

(ف) $(\Theta \Theta$ (C) 2020 submitted by the authors. Open access publication under the terms and conditions of the Creative Commons Attribution (CC- BYNC- ND) license. (https://creativecommons.org/licenses/by-nc-nd/2.0/). 